\section{Extended interactive data manipulation and analysis}

\author{
SAM HOLLINGSWORTH and G. C. JERNSTEDT \\ Darmouth College', Hanover. New Hampshire 03755
}

A large proportion of the data collected in the social sciences are in the form of a series of numbers or alphabetic characters that represent information about individual subjects. Sources of such information include questionnaires, surveys. psychodiagnostic devices such as personality inventories, objective examinations in a course. and, obviously. experimental investigations. The basic analises of these classes of data are remarkably similar. and the packige of progranis described here provides a general-purpose means of manipulating and analyzing such data.

Description. The programs treat a body of data by first casting it into a master matrix. This matrix contains one row for each subject and one columu for each score. Cell i.j thus contains the $j$-th score for the $i-t h$ subject. Each program in the package operates on this matrix. and all manipulations of the data are in the form of inputs wor outputs from the matrix. To ensure the full archival value of the data. labels that document the contents of each cell are automatically stored with the matrix.

Specitied portions of a master matrix may be transformed in various ways. Preprogrammed transformations for analysis of variance include logarithmic. trigonometric. reciprocal, and square root functions. Furthermore. the user may supply special transtormation keys to convert a raw score to a standardized score, such as a population percentile.

Input. Data may be entered into the master matrix from any peripheral device. The package presently accepts data from punched cards. magnetic tape, or any Teletype-format input terminal. The first program of the package asks a series of questions that allows the investigator to specify the exact format of the data. The format is entirely open and may involve indicating the separation of individual items of data by spaces. by any alphanumeric character, or by position on the input record. The package also includes a program that checks for the required number of data points. and which will. if the user wishes. replace a missing datum with the mean for that item averaged across the other subjects in that group.

Some applications. such as the scoring of psychodiagnostic tests or course examinations. will require the preparation of an answer kes. Decumentation that is included with the package describes the format of such kess at length.

This project was supported in part by a grant from Outward Bound. Inc.. and by funds from the Dartmouth Outward Bound Center.
Output. The simplest output is a tabular listing of the master matrix. This is of use for the storage of data. The user may request a listing of the means and standard deviations of the columns of the master matrix. Column means may also be conputed for user-specitied subgroups of subjects. $\dot{A}$ program is included that provides a complete correlation matrix of any specitied portion of the data. In general. the output of the package is compatible with the input requirements of standard nonparametric statistical programs and multivariate analysis programs. although these have not been implemented into the package.

A program has been prepared that facilitates the separation of the data into various subgroups. That is, subjects may be grouped according to particular treatments or according to their responses on particular itens. The data may then be analyzed by a broad-range analysis of variance program. included in the package, that has been described by Jernstedt (1972). The analysis of variance design may involve up 10 three between factors and one within factor. and there may be unequal numbers of subjects in each group. In addition to the suns of squares, degrees of freedom. and $F$ ratios. the program also calculates and prints the exact probability of the $\mathrm{F}$ ratio and the proportion of variance accounted for by each factor. Newnan-Keuls tests of differences between individual means may also be performed.

Use. The package has been used in its present form to score course examinations and produce an item analysis on the test questions, to score and report to patients the results of a battery of standard psychological tests. to score and analyze a series of questionnaires in a longitudinal study of attitude change, to interrelate and explore a number of sets of data in order to predict performances in a large selt-paced course, to build a data bank of and analyze course evaluations for an academic department. to tabulate and analyze a wide variety of data measures in an animal learning experiment, and to analyze the results of a psychophysiological experiment involving the digitizing of analog data.

Computer and language. The prograns are written in BASIC. Sharpe $(1968)$ describes a FORTRAN progran that modities BASIC programs for use on FORTRAN-based systems. The programs in the package have been used on a Honeywell 635 system in both the interactive time-sharing mode and in the batch processing mode. As the programs are generally quite compact. their use in small computers is limited only by the size of the data base.

Availability. A listing of the programs and a manual describing their use may be obtained from the second author.

\section{REFERENCES}

Jernstedt. G. C. Broad-range analysis of variance. Behavioral Science. 1972, 19. 397-398.

SHARPE, W. F. UWBIC-University of Washington BASIC inter pretive compiler. Behavioral Science, 1968,13,81. 